Bull. Korean Math. Soc. 46 (2009), No. 6, pp. 1051-1060

DOI 10.4134/BKMS.2009.46.6.1051

\title{
AN IDEAL-BASED ZERO-DIVISOR GRAPH OF 2-PRIMAL NEAR-RINGS
}

\author{
Patchirajulu Dheena and Balasubramanian Elavarasan
}

\begin{abstract}
In this paper, we give topological properties of collection of prime ideals in 2-primal near-rings. We show that $\operatorname{Spec}(N)$, the spectrum of prime ideals, is a compact space, and $\operatorname{Max}(N)$, the maximal ideals of $N$, forms a compact $T_{1}$-subspace. We also study the zero-divisor graph $\Gamma_{I}(R)$ with respect to the completely semiprime ideal $I$ of $N$. We show that $\Gamma_{\mathbb{P}}(R)$, where $\mathbb{P}$ is a prime radical of $N$, is a connected graph with diameter less than or equal to 3 . We characterize all cycles in the graph $\Gamma_{\mathbb{P}}(R)$.
\end{abstract}

\section{Preliminaries}

In [3], Beck introduced the concept of a zero-divisor graph of a commutative ring with identity, but this work was mostly concerned with coloring of rings. In [2], Anderson and Livingston associated a graph (simple) $\Gamma(R)$ to a commutative ring $R$ with identity with vertices $Z(R)^{*}=Z(R) \backslash\{0\}$, the set of nonzero zero-divisor of $R$, and for distinct $x, y \in Z(R)^{*}$, the vertices $x$, and $y$ are adjacent if and only if $x y=0$. They investigated the interplay between the ring-theoretic properties of $R$ and the graph-theoretics properties of $\Gamma(R)$.

In [9], Redmond has generalized the notion of the zero-divisor graph. For a given ideal $I$ of $R$, he defined an undirected graph $\Gamma_{I}(R)$ with vertices $\{x \in$ $R \backslash I: x y \in I$ for some $y \in R \backslash I\}$, where distinct vertices $x$ and $y$ are adjacent if and only if $x y \in I$.

In this paper, we study the undirected graph $\Gamma_{I}(N)$ of near-rings for any completely semiprime ideal $I$ of $N$. We extend the results obtained by K. Samei [11] for reduced rings to 2-primal near-rings. Clearly, reduced rings are 2-primal near-rings.

Let $N$ be a near-ring with identity. Let $J$ be a completely semiprime ideal of $N$. The zero-divisor graph of $N$ with respect to the ideal $J$, denoted by $\Gamma_{J}(N)$, is the graph whose vertices are the set $\{x \in N \backslash J: x y \in J$ for some $y \in N \backslash J\}$ with distinct vertices $x$ and $y$ are adjacent if and only if $x y \in J$. If $J=0$, then

Received November 1, 2007; Revised July 13, 2009.

2000 Mathematics Subject Classification. 16Y30, 13A99.

Key words and phrases. graph, prime ideal, 2-primal, Zariski topology and cycle. 
$\Gamma_{J}(N)=\Gamma(N)$, and $J$ is a non-zero completely prime ideal of $N$ if and only if $\Gamma_{J}(N)=\phi$.

Example 1.1. Let $N=\left(\begin{array}{cc}F & F \\ 0 & F\end{array}\right)$, where $F=\{0,1\}$ is the field under addition and multiplication modulo 2. Then its prime radical $P=\left\{\left(\begin{array}{ll}0 & 0 \\ 0 & 0\end{array}\right),\left(\begin{array}{ll}0 & 1 \\ 0 & 0\end{array}\right)\right\}$ is a completely reflexive ideal of the near-ring $N$ and its ideal based zero-divisor $\operatorname{graph} \widehat{\Gamma_{P}(N)}$ is:

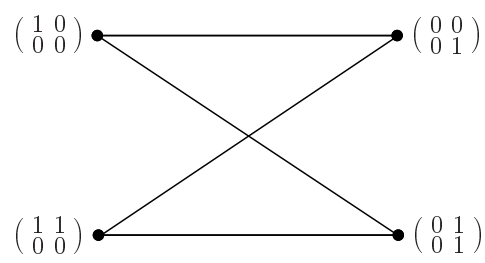

Remark 1.2. In the above example, $N$ is a 2-primal near-ring, but neither reduced nor commutative.

Throughout this paper $N$ is a zero symmetric near-ring with identity unless otherwise stated, and its prime radical is not a prime ideal of $N$.

Let $\mathbb{P}$ denote the prime radical, and let $N(N)$ denote the set of nilpotent elements of $N$. For any vertices $x, y$ in a graph $G$, if $x$ and $y$ are adjacent, we denote it as $x \approx y$. A near-ring $N$ is called a 2-primal if $\mathbb{P}=N(N)$. A near-ring $N$ is said to be reduced if $N(N)=0$. Clearly, reduced near-rings are 2-primal, but the converse need not be true (See Example 1.3 of [5]). A near-ring $N$ is called pm if each prime ideal in $N$ is contained in a unique maximal ideal of $N$.

We use $\operatorname{Spec}(N), \operatorname{Max}(N)$, and $\operatorname{Min}(N)$ for the spectrum of prime ideals, maximal ideal and minimal prime ideals of $N$, respectively.

For any ideal $J$ of $N$ and $a \in N$, we define $V(a)=\{P \in \operatorname{Spec}(N): a \in P\}$ and $D(J)=\operatorname{Spec}(N) \backslash V(J)$. Let $V(J)=\cap_{a \in J} V(a)$. Then $F=\{V(J): J$ is an ideal of $N\}$ is closed under finite union and arbitrary intersections, so that there is a topology on $\operatorname{Spec}(N)$ for which $F$ is the family of closed sets. This is called the Zariski topology. Note that $V(A)=(\langle J\rangle)$ for any subset $A$ of $N$. Let $\mathcal{B}=\{D(a): a \in N\}$. Then $\mathcal{B}$ is a basis for a topology on $\operatorname{Spec}(N)$.

The operations $c l$ and int denote the closure and the interior in $\operatorname{Spec}(N)$. We also set $V^{\prime}(a)=V(a) \cap \operatorname{Min}(N) ; D^{\prime}(a)=D(a) \cap \operatorname{Min}(N)$.

For any subset $S$ of $N$, we define $\mathbb{P}_{S}=\{n \in N: n S \subseteq \mathbb{P}\}$. We set $\operatorname{Supp}(a)=$ $\cap_{x \in \mathbb{P}_{a}} V(x)$.

For distinct vertices $x$ and $y$ of $\Gamma_{\mathbb{P}}(N)$, let $d(x, y)$ be the length of the shortest path from $x$ to $y$. The diameter of a connected graph is the supremum of the distances between vertices. The associated number $e(a)$ for a vertex $a$ in $\Gamma_{\mathbb{P}}(R)$ is defined by $e(a)=\max \{d(a, b): a \neq b\}$.

A graph $G$ is called triangulated (hyper-triangulated) if each vertex (edge) of $G$ is a vertex (edge) of a triangle. 
A point $P$ of $\operatorname{Spec}(N)$ is said to be quasi-isolated if $P$ is a minimal prime ideal and $P$ is not contained in the union of all minimal prime ideals of $N$ different from $P$.

If $a$ and $b$ are the two vertices in $\Gamma_{\mathbb{P}}(N)$, by $c(a, b)$ we mean the length of the smallest cycle containing $a$ and $b$. For every two vertices $a$ and $b$, all possible cases for $c(a, b)$ are given in Theorem 3.9. In this paper the notations of graph theory are from [4], the notations of near-ring are from [8], and the notations of topology are from [6] and [7].

\section{Topological space of $\operatorname{Spec}(N)$}

In this section, we associate the near-ring properties of $N$ and the topological properties of $\operatorname{Spec}(N)$. We start this section with the following useful lemma.

Lemma 2.1. Let $N$ be a near-ring. If $A$ is a subset of $\operatorname{Spec}(N)$, then there exists an ideal $J=\cap A$ of $N$ with $c l(A)=V(J)$. In particular, if $A$ is a closed subset of $\operatorname{Spec}(N)$, then $A=V(J)$ for some ideal $J$ of $N$.

Proof. Let $P_{1} \in V(J)$ and let $D(x)$ be any arbitrary element in $\mathcal{B}$ such that $P_{1} \in D(x)$. Suppose that $D(x) \cap A=\phi$. Then $x \in J$, and so $P_{1} \in V(x)$, a contradiction. Thus $D(x) \cap A \neq \phi$, and hence, the result follows from Theorem 17.5 of $[7]$.

In view of above lemma, we have the following remarks.

Remark 2.2. Let $N$ be a near-ring.

(i) The closure of $P \in \operatorname{Spec}(N)$ is $V(P)$.

(ii) A point $P \in \operatorname{Spec}(N)$ is closed if and only if $P \in \operatorname{Max}(N)$.

(iii) If $P, Q \in \operatorname{Spec}(N)$ with $\operatorname{cl}(P)=\operatorname{cl}(Q)$, then $P=Q$.

With the help of Lemma 2.1, we have the following some important characterizations of $\operatorname{Spec}(N)$.

Theorem 2.3. Let $N$ be a near-ring.

(i) If $F \subseteq \operatorname{Spec}(N)$ is a closed set and $D(K)$ is an open set in $\operatorname{Spec}(N)$ satisfying $F \cap \operatorname{Max}(N) \subseteq D(K)$, then $F \subseteq D(K)$.

(ii) $\operatorname{Spec}(N)$ is a compact space.

(iii) $\operatorname{Max}(N)$ is a compact $T_{1}$ subspace.

(iv) If $\operatorname{Spec}(N)$ is normal, then $\operatorname{Max}(N)$ is a Hausdorff space.

(v) If $\mathbb{P}=\cap \operatorname{Max}(N)$ and $\operatorname{Max}(N)$ is a Hausdorff space, then $\operatorname{Spec}(N)$ is normal.

Proof. (i) Suppose that there is $P \in F$ with $P \notin D(K)$. Then $K+L \subseteq P$ since $F=V(L)$ for some ideal $L$ of $N$. Hence, each maximal ideal $M$ containing $P$ is also in $F$. Then $M \in F \cap \operatorname{Max}(N)$, and so $M \in D(K)$, a contradiction.

(ii) Let $\mathcal{B}=\left\{D\left(s_{i}\right): s_{i} \in J\right\}$ be the basis of $N$, for any subset $J$ of $N$, and suppose that $\operatorname{Spec}(N)=\cup_{j \in J} D\left(s_{j}\right)$. Then $\phi=\cap_{j \in J}\left(\operatorname{Spec}(N) \backslash D\left(s_{j}\right)\right)=$ $\cap_{j \in J} V\left(s_{j}\right)=V\left(\left\langle s_{j} ; j \in J\right\rangle\right)=V\left(\sum_{j \in J}\left\langle s_{j}\right\rangle\right)$ which gives $\sum_{j \in J}\left\langle s_{j}\right\rangle=N$. Then 
there exists $K \subset J$ finite with $1=\sum_{k \in K} s_{k}^{\prime}$, where $s_{k}^{\prime} \in\left\langle s_{k}\right\rangle$ which implies $\operatorname{Spec}(N)=\cup_{k \in K} D\left(s_{k}^{\prime}\right)$. Indeed, clearly $\cup_{k \in K} D\left(s_{k}^{\prime}\right) \subseteq \operatorname{Spec}(N)$ and $\operatorname{suppose}$ $P \in \operatorname{Spec}(N)$ with $P \notin \cup_{k \in K} D\left(s_{k}^{\prime}\right)$. Then $s_{k}^{\prime} \in P$ for all $k \in K$ which implies $1 \in P$, a contradiction. Hence $\operatorname{Spec}(N)$ is a compact space.

(iii) Let $\mathcal{B}=\left\{D\left(s_{i}\right): s_{i} \in J\right\}$ be the basis of $N$, for any subset $J$ of $N$, and suppose that $\operatorname{Max}(N)=\left(\cup_{i \in J} D\left(s_{i}\right)\right) \cap \operatorname{Max}(N)$. Then

$$
\begin{aligned}
\phi=\cap_{i \in J}\left(\operatorname{Max}(N) \backslash D\left(s_{i}\right)\right) & =\left(\cap_{i \in J} V\left(s_{i}\right)\right) \cap \operatorname{Max}(N) \\
& =V\left(\sum_{i \in I}\left\langle s_{i}\right\rangle\right) \cap \operatorname{Max}(N)
\end{aligned}
$$

which imply $\sum_{i \in J}\left\langle s_{i}\right\rangle=N$. Then there exists $J_{1} \subset J$ finite with $1=\sum_{j \in J_{1}} s_{j}$, and so $\operatorname{Max}(N)=\cup_{j \in J_{1}} D\left(s_{j}\right)$.

Let $M_{1}$ and $M_{2}$ be two distinct elements in $\operatorname{Max}(N)$. Then $M_{1} \in D\left(M_{2}\right)$ and $M_{2} \in D\left(M_{1}\right)$, and so $\operatorname{Max}(N)$ is a $T_{1}$ space.

(iv) Let $M_{1}$ and $M_{2}$ be distinct elements in $\operatorname{Max}(N)$. Then $\left\{M_{1}\right\}$ and $\left\{M_{2}\right\}$ are closed subsets in both $\operatorname{Spec}(N)$ and $\operatorname{Max}(N)$. If $\operatorname{Spec}(N)$ is normal, then there exist disjoint open sets $D(I)$ and $D(J)$ such that $\left\{M_{1}\right\} \subseteq D(I)$ and $\left\{M_{2}\right\} \subseteq D(J)$ for some ideals $I$ and $J$ of $N$, respectively. So, $\bar{M}_{1} \in D(I) \cap$ $\operatorname{Max}(N)$, and $M_{2} \in D(J) \cap \operatorname{Max}(N)$, which imply $\operatorname{Max}(N)$ is a Hausdorff space.

(v) Let $F_{1}$ and $F_{2}$ be two disjoint closed subsets of $\operatorname{Spec}(N)$. Then $F_{1} \cap$ $\operatorname{Max}(N)$ and $F_{2} \cap \operatorname{Max}(N)$ are also disjoint subsets of $\operatorname{Max}(N)$. By Theorem 32.3 in [7], $\operatorname{Max}(N)$ is normal. So, there are open subsets $D(J)$ and $D\left(J_{1}\right)$ of $\operatorname{Spec}(N)$ such that $F_{1} \cap \operatorname{Max}(N) \subseteq A, F_{2} \cap \operatorname{Max}(N) \subseteq B$ and $A \cap B=\phi$, where $A=D(J) \cap \operatorname{Max}(N)$ and $B=D\left(J_{1}\right) \cap \operatorname{Max}(N)$.

Assume $\mathbb{P}=\cap \operatorname{Max}(N)$. Then $J J_{1} \subseteq \cap \operatorname{Max}(N)=\mathbb{P}$ since $D(J) \cap D\left(J_{1}\right)=$ $D\left(J J_{1}\right)$, and so $D(J) \cap D\left(J_{1}\right)=\phi$. By (i), we have $F_{1} \subseteq D(J)$ and $F_{2} \subseteq$ $D\left(J_{1}\right)$.

Theorem 2.4. Let $N$ be a 2-primal near-ring. Then $\mathbb{P}_{S}=\cap V\left(\mathbb{P}_{S}\right)$ for any subset $S$ of $N$.

Proof. Clearly, $\mathbb{P}_{S} \subseteq \cap V\left(\mathbb{P}_{S}\right)$. Let $a \in N \backslash \mathbb{P}_{S}$. Then as $\notin P$ for some $P \in$ $\operatorname{Spec}(N)$ and $s \in S$ which implies $\mathbb{P}_{S} \subseteq P$. Thus, $a \notin P \in V\left(\mathbb{P}_{S}\right)$, and hence, $\cap V\left(\mathbb{P}_{S}\right) \subseteq \mathbb{P}_{S}$

Lemma 2.5. Let $N$ be a 2-primal near-ring and let $a, b \in N$. Then int $V(a) \subseteq$ int $V(b)$ if and only if $\mathbb{P}_{a} \subseteq \mathbb{P}_{b}$.

Proof. Let int $V(a) \subseteq$ int $V(b)$ for any $a, b \in N$ and let $x \in \mathbb{P}_{a}$. Then $\operatorname{Spec}(N) \backslash V(x) \subseteq$ int $V(a) \subseteq$ int $V(b) \subseteq V(b)$, which gives $b x \in \mathbb{P}$, so $x \in \mathbb{P}_{b}$.

Conversely, let $\mathbb{P}_{a} \subseteq \mathbb{P}_{b}$ and let $P \in$ int $V(a)$. Suppose $P \notin V(b)$. By Lemma 2.1, if $P \notin \operatorname{Spec}(N) \backslash$ int $V(a)$, then there is $0 \neq c \in N$ with $\operatorname{Spec}(N) \backslash$ int $V(a) \subseteq$ $V(c)$ and $c \notin P$. Clearly $a c \in \mathbb{P}$ and $b c \notin \mathbb{P}$. Then $c \in \mathbb{P}_{a}$ and $c \notin \mathbb{P}_{b}$, a contradiction. 
Lemma 2.6. Let $N$ be a 2-primal near-ring. Then for every $a \in N, \operatorname{cl}(D(a))=$ $V\left(\mathbb{P}_{a}\right)=\operatorname{Supp}(a)=\operatorname{Spec}(N) \backslash$ int $V(a)$.

Proof. Let $a \in N, P \in V\left(\mathbb{P}_{a}\right)$, and let $D(x)$ be any arbitrary basis element in $\mathcal{B}$ such that $P \in D(x)$. Let $P \notin D(a)$ and suppose $D(a) \cap D(x)=\phi$. Then $D(x a) \subseteq D(x) \cap D(a)=\phi$, and so $x a \in \mathbb{P}$ which implies $x \in P$, a contradiction. Thus, $\bar{D}(a) \cap D(x) \neq \phi$, and hence, $V\left(\mathbb{P}_{a}\right)=\operatorname{cl}(D(a))$.

Let $P \in \operatorname{cl}(D(a))$ and suppose that $P \in$ int $V(a)$. Then there exists an open set $U$ of $\operatorname{Spec}(N)$ with $P \in U \subseteq V(a)$, and so $P \notin \operatorname{Spec}(N) \backslash U$, a contradiction. Let $P \in \operatorname{Spec}(N) \backslash$ int $V(a)$ and let $D(x)$ be any arbitrary element in $\mathcal{B}$ with $P \in D(x)$. Suppose that $D(x) \cap D(a)=\phi$. Then $P \in D\left(\mathbb{P}_{a}\right) \subseteq V(a)$, a contradiction.

The following result gives the condition under which a subset of $\operatorname{Spec}(N)$ of 2-primal near-ring to be clopen, which will be used in our main result in Section 3.

Lemma 2.7. Let $N$ be a 2-primal near-ring. Then $A$ is a clopen subset of $\operatorname{Spec}(N)$ if and only if there exists an element $a \in N$ with $a \in P$ or $-1+a \in P$ for all $P \in \operatorname{Spec}(N)$ and $A=V(a)$.

Proof. Suppose that $A$ is a clopen subset of $\operatorname{Spec}(N)$. Let $J=\cap A$ and $J_{1}=$ $\cap A^{c}$. Then by Lemma $2.1 A=\operatorname{cl}(A)=V(J)$ and $A^{c}=V\left(J_{1}\right)$. So, $V(J) \cap$ $V\left(J_{1}\right)=\phi$, which gives $J+J_{1}=N$. Then there exists $a \in J$ and $a^{\prime} \in J_{1}$ such that $a+a^{\prime}=1$. Therefore $a(-1+a) \in \mathbb{P}$. Thus, for every prime ideal $P$, we have $a \in P$ or $-1+a \in P$. Consequently, $A=V(J)=V(a)$. The converse is trivial.

Theorem 2.8. Let $N$ be a 2-primal and pm near-ring. Then $\operatorname{Max}(N)$ is a compact Hausdorff space.

Proof. By Lemma 2.3(iii), $\operatorname{Max}(N)$ is a compact space. Let $M_{1}, M_{2} \in \operatorname{Max}(N)$ and consider the multiplicative subset

$$
S=\left\{a_{1} b_{1} \cdots a_{n-1} b_{n-1} a_{n} b_{n}: a_{i} \notin M_{1}, b_{i} \notin M_{2}, n, i \in\{1,2, \ldots, n\}\right\} .
$$

Suppose that $0 \notin S$. Then there is a prime ideal $P$ of $N$ with $P \cap S=\phi$ and hence $P \subseteq M_{1} \cap M_{2}$, a contradiction. So, there exist $a_{i} \notin M_{1}$ and $b_{i} \notin M_{2}$ such that $a_{1} b_{1} \cdots a_{n} b_{n}=0$. We now have elements $x_{1} \notin M_{1}$ and $x_{2} \notin M_{2}$ with $x_{1} x_{2} \in \mathbb{P}$, which imply $D\left(x_{1}\right)$ and $D\left(x_{2}\right)$ are disjoint with $M_{1} \in D\left(x_{1}\right)$ and $M_{2} \in D\left(x_{2}\right)$.

The following is an immediate corollary of Theorem 2.8 .

Corollary 2.9 ([12], Lemma 2.1). If $R$ is a 2-primal and pm ring, then $\operatorname{Max}(R)$ is a compact Hausdorff space. 


\section{Distance and cycles in $\Gamma_{\mathbb{P}}(N)$}

In this section, we associate the near-ring properties of $N$ and the graph properties of $\Gamma_{\mathbb{P}}(N)$.

Theorem 3.1. Let $N$ be a 2-primal near-ring. Then $\Gamma_{\mathbb{P}}(N)$ is connected and $\operatorname{diam} \Gamma_{\mathbb{P}}(N) \leq 3$.

Proof. Let $x, y \in \Gamma_{\mathbb{P}}(N)$ be distinct. If $x y \in \mathbb{P}$, then $d(x, y)=1$. Otherwise, there are $a, b \in N \backslash(\mathbb{P} \cup\{x, y\})$ such that $a x, b y \in \mathbb{P}$.

If $a=b$, then $x \approx a \approx y$ is a path of length 2 . Thus, we assume that $a \neq b$. If $a b \in \mathbb{P}$, then $x \approx a \approx b \approx y$ is a path of length 3 ; and hence $d(x, y) \leq 3$. Otherwise, $x \approx a b \approx y$ is a path of length 2 ; thus, $d(x, y)=2$. Hence, $d(x, y) \leq 3$.

Lemma 3.2. Let $N$ be a 2-primal near-ring and let $a, b \in \Gamma_{\mathbb{P}}(N)$. Then

(i) $\operatorname{Supp}(a) \cup \operatorname{Supp}(b) \neq \operatorname{Spec}(N)$ if and only if $\operatorname{Supp}(a) \cup \operatorname{Supp}(b) \subseteq V(c)$ for some $c \in \Gamma_{\mathbb{P}}(N)$.

(ii) $D(a) \cap D(b) \neq \phi$ if and only if there exists $c \in \Gamma_{\mathbb{P}}(N)$ such that $\phi \neq$ $D(a) \cap D(b) \subseteq V(c)$.

Proof. (i) Suppose $\operatorname{Supp}(a) \cup \operatorname{Supp}(b) \neq \operatorname{Spec}(N)$. Then there exists an element $P \in \operatorname{Spec}(N)$ with $x, y \notin P$ for some $x \in \mathbb{P}_{a}$ and $y \in \mathbb{P}_{b}$. So, $x y \notin \mathbb{P}$. It is easy to see that $\operatorname{Supp}(a) \cup \operatorname{Supp}(b) \subseteq V(x y)$.

Conversely, suppose that $\operatorname{Supp}(a) \cup \operatorname{Supp}(b)=\operatorname{Spec}(N)$. Then $c \in \mathbb{P}$, a contradiction. Hence, $\operatorname{Supp}(a) \cup \operatorname{Supp}(b) \neq \operatorname{Spec}(N)$.

(ii) Straightforward.

Now by Theorem 3.1, and Lemma 3.2, we have the following characterizations of the diameter of $\Gamma_{\mathbb{P}}(N)$.

Theorem 3.3. Let $N$ be a 2-primal near-ring and let $a, b \in \Gamma_{\mathbb{P}}(N)$ be distinct elements. Then

(i) For any $c \in \Gamma_{\mathbb{P}}(N)$, we have $c$ is adjacent to both $a$ and $b$ if and only if $\operatorname{Supp}(a) \cup \operatorname{Supp}(b) \subseteq V(c)$.

(ii) $d(a, b)=1$ if and only if $D(a) \cap D(b)=\phi$.

(iii) $d(a, b)=2$ if and only if $D(a) \cap D(b) \neq \phi$ and $\operatorname{Supp}(a) \cup \operatorname{Supp}(b) \neq$ $\operatorname{Spec}(N)$.

(iv) $d(a, b)=3$ if and only if $D(a) \cap D(b) \neq \phi$ and $\operatorname{Supp}(a) \cup \operatorname{Supp}(b)=$ $\operatorname{Spec}(N)$.

Proof. (i) Let $c \in \Gamma_{\mathbb{P}}(N)$. Then $c$ is adjacent to both $a$ and $b$ if and only if $D(a) \cap D(c)=D(b) \cap D(c)=\phi$ if and only if $\operatorname{Supp}(a) \cup \operatorname{Supp}(b) \subseteq V(c)$.

(ii) Trivial.

(iii) Let $a, b \in \Gamma_{\mathbb{P}}(N)$. Then $d(a, b)=2$ if and only if $a b \notin \mathbb{P}$ and there exists $c \in \Gamma_{\mathbb{P}}(N)$ such that $c$ is adjacent to both $a$ and $b$ if and only if $D(a) \cap$ $D(b) \neq \phi$ and $\operatorname{Supp}(a) \cup \operatorname{Supp}(b) \subseteq V(c)$ if and only $D(a) \cap D(b) \neq \phi$ and $\operatorname{Supp}(a) \cup \operatorname{Supp}(b) \neq \operatorname{Spec}(N)$ by Lemma 3.2 . 
(iv) By Theorem 3.1, $d(a, b)=3$ if and only if $d(a, b) \neq 1,2$ if and only if $D(a) \cap D(b) \neq \phi$ and $\operatorname{Supp}(a) \cup \operatorname{Supp}(b)=\operatorname{Spec}(N)$ by (i) and (ii).

Since the reduced commutative ring is also a 2-primal near-ring, the following corollary is immediate.

Corollary 3.4 ([11], Proposition 2.2). Let $R$ be a commutative reduced ring and let $a, b, c \in \Gamma(R)$ be distinct elements. Then

(i) $c$ is adjacent to both $a$ and $b$ if and only if $\operatorname{Supp}(a) \cup \operatorname{Supp}(b) \subseteq V(c)$.

(ii) $d(a, b)=1$ if and only if $D(a) \cap D(b)=\phi$.

(iii) $d(a, b)=2$ if and only if $D(a) \cap D(b) \neq \phi$ and $\operatorname{Supp}(a) \cup \operatorname{Supp}(b) \neq$ $\operatorname{Spec}(R)$.

(iv) $d(a, b)=3$ if and only if $D(a) \cap D(b) \neq \phi$ and $\operatorname{Supp}(a) \cup \operatorname{Supp}(b)=$ $\operatorname{Spec}(R)$.

The following theorem shows that every minimal prime ideal of 2-primal near-ring that doesn't contain both $a$ and $\mathbb{P}_{a}$ for any $a \in N$.

Theorem 3.5. Let $N$ be a 2-primal near-ring and let $a \in N$. Then $V^{\prime}(a)=$ $D^{\prime}\left(\mathbb{P}_{a}\right)$ and $D^{\prime}(a)=V^{\prime}\left(\mathbb{P}_{a}\right)$. In particular, $V^{\prime}(a)$ and $V^{\prime}\left(\mathbb{P}_{a}\right)$ are disjoint clopen subsets of $\operatorname{Spec}(N)$. Also, $\operatorname{Min}(N)$ is a Hausdorff space.

Proof. Let $P \in V^{\prime}(a)$ and suppose $P \notin D^{\prime}\left(\mathbb{P}_{a}\right)$. Let $M=\left\{a, a^{2}, \ldots\right\}$ be multiplicative closed system and let $S=\{I \unlhd N: I \subseteq P$ and $I \cap M=\phi\}$. Since $\mathbb{P}_{a} \in S, S \neq \phi$. Then by Zorn's Lemma, there exists a maximal ideal $\bar{P}$ in $S$ with $\bar{P} \subseteq P$ and $\bar{P} \cap M=\phi$. Let $J$ and $J_{1}$ be ideals of $N$ such that $\bar{P} \subset J$ and $\bar{P} \subset J_{1}$.

Case (i): If $P \subset J$ and $P \subset J_{1}$, then $J J_{1} \not \subset P$. So $J J_{1} \nsubseteq \bar{P}$.

Case (ii): If $J \subseteq P$ and $J_{1} \subseteq P$, then $J \cap M \neq \phi$ and $J_{1} \cap M \neq \phi$. Then there exist $j \in J \cap M$ and $j_{1} \in J_{1} \cap M$ with $j^{\prime} j_{1}^{\prime} \in M$ for some $j^{\prime} \in J$ and $j_{1}^{\prime} \in J_{1}$, which gives $J J_{1} \cap M \neq \phi$. So, $J J_{1} \nsubseteq \bar{P}$.

Case (iii): If $J \subseteq P$ and $P \subset J_{1}$, then by Case (ii), we have $J P \nsubseteq \bar{P}$. So $J J_{1} \nsubseteq \bar{P}$.

Thus, $\bar{P}$ is a prime ideal with $\bar{P} \subset P$, contradicting the minimality of $P$. Hence, $V^{\prime}(a)=D^{\prime}\left(\mathbb{P}_{a}\right)$. Similarly, we have $D^{\prime}(a)=V^{\prime}\left(\mathbb{P}_{a}\right)$.

Let $P \neq P^{\prime} \in \operatorname{Min}(N)$ and $a \in P \backslash P^{\prime}$. Then $V^{\prime}(a)$ and $V^{\prime}\left(\mathbb{P}_{a}\right)$ are disjoint open sets containing $P$ and $P^{\prime}$, respectively.

Lemma 3.6. Let $N$ be a 2-primal near-ring and let $a \in \Gamma_{\mathbb{P}}(N)$. If $e(a)=1$, then $\mathbb{P}_{a}$ is a completely prime ideal of $N$.

Proof. Straightforward.

Theorem 3.7. Let $N$ be a 2-primal near-ring and $2 \notin \mathbb{P}$. Then

(i) $\Gamma_{\mathbb{P}}(N)$ is a triangulated graph if and only if $\operatorname{Spec}(N)$ has no quasiisolated points. 
(ii) $\Gamma_{\mathbb{P}}(N)$ is a hyper-triangulated graph if and only if $\operatorname{Spec}(N)$ is connected space and for any $a, b \in \Gamma_{\mathbb{P}}(N)$, we have that $a b \in \mathbb{P}$ and $D(a) \cup D(b) \neq$ $\operatorname{Spec}(N)$ imply $\operatorname{Supp}(a) \cup \operatorname{Supp}(b) \neq \operatorname{Spec}(N)$.

(iii) If $2 \notin \Gamma_{\mathbb{P}}(N)$, then every vertex of $\Gamma_{\mathbb{P}}(N)$ is a 4 -cycle vertex.

Proof. (i) Let $\Gamma_{\mathbb{P}}(N)$ be a triangulated graph and $\operatorname{suppose} \operatorname{Spec}(N)$ has a quasiisolated point $P$. Then $D^{\prime}\left(\mathbb{P}_{a}\right)=V^{\prime}(a)=\{P\}$ for some $a \in P$. Clearly, $a \in$ $\Gamma_{\mathbb{P}}(N)$, and since $\Gamma_{\mathbb{P}}(N)$ is a triangulated graph, there are $b, c \in \Gamma_{\mathbb{P}}(N)$ such that $a b, a c, b c \in \mathbb{P}$. Thus, $D^{\prime}(a) \subseteq V^{\prime}(b)$, and $\phi \neq D^{\prime}(c) \subseteq V^{\prime}(a) \cap V^{\prime}(b)=\{P\}$, which gives $V^{\prime}(b)=\operatorname{Min}(N)$, a contradiction. Hence, $\operatorname{Spec}(N)$ does not contain quasi-isolated points.

Conversely, suppose that $\operatorname{Spec}(N)$ does not contain quasi-isolated points and take $a \in \Gamma_{\mathbb{P}}(N)$. Then there are two different points $P, P^{\prime} \in V^{\prime}(a)=D^{\prime}\left(\mathbb{P}_{a}\right)$. Since $\mathbb{P}_{a} \nsubseteq P^{\prime}$, there exists $z \in \mathbb{P}_{a}$ such that $z \notin P^{\prime}$. Also, there exists $y \in P$ with $y \notin P^{\prime}$. Clearly, $z y \notin \mathbb{P}$ and $P \in V^{\prime}(z y)=D^{\prime}\left(\mathbb{P}_{z y}\right)$, which imply $P \notin$ $\operatorname{Supp}(z y)$. Thus $\operatorname{Supp}(a) \cup \operatorname{Supp}(z y) \neq \operatorname{Spec}(N)$. Then by Lemma 3.2, there exists $c \in \Gamma_{\mathbb{P}}(N)$ such that $\operatorname{Supp}(a) \cup \operatorname{Supp}(z y) \subseteq V(c)$, so by Theorem 3.3 (i), $c$ is adjacent to both $a$ and $z y$.

(ii) Let $\Gamma_{\mathbb{P}}(N)$ be a hyper-triangulated graph. If $\operatorname{Spec}(N)$ is not connected, then by Lemma 2.7, there exists an element $a \in \Gamma_{\mathbb{P}}(N)$. Since $\operatorname{Supp}(a) \cup$ $\operatorname{supp}(-1+a)=\operatorname{Spec}(N)$, by Theorem 3.3, there is no vertex adjacent to both $a$ and $-1+a$, a contradiction. The second part follows from Lemma 3.2 and Theorem 3.3.

Conversely, let $a \approx b$ be an edge in $\Gamma_{\mathbb{P}}(N)$. Since $D(a) \cap D(b)=\phi$ and $\operatorname{Spec}(N)$ is connected, $D(a) \cup D(b) \neq \operatorname{Spec}(N)$. Thus by hypothesis, $\operatorname{Supp}(a) \cup$ $\operatorname{Supp}(b) \neq \operatorname{Spec}(N)$. Therefore, by Lemma 3.2 and Theorem 3.3, there exists a vertex adjacent to both $a$ and $b$.

(iii) Let $a \in \Gamma_{\mathbb{P}}(N)$. Then there exists $b \in N \backslash \mathbb{P}$ such that $a b \in \mathbb{P}$. Since $2 \notin \Gamma_{\mathbb{P}}(N)$, we have $2 a \neq b$ and $a \neq 2 b$. So $a, b, 2 a$ and $2 b$ are all distinct. Also, $a b,(2 a) b,(2 a)(2 b)$ and $a(2 b)$ belong to $\mathbb{P}$. Hence $a, b, 2 a$ and $2 b$ is a cycle with length 4 containing $a$.

As an immediate application of Theorem 3.7, we have the following corollary.

Corollary 3.8 ([11], Theorem 3.1). Let $R$ be a commutative reduced ring. Then

(i) $\Gamma(R)$ is a triangulated graph if and only if $\operatorname{Spec}(R)$ has no quasi-isolated points.

(ii) $\Gamma(R)$ is a hyper-triangulated graph if and only if $\operatorname{Spec}(R)$ is connected space and for any $a, b \in \Gamma(R)$, we have that $a b \in \mathbb{P}$ and $D(a) \cup D(b) \neq$ $\operatorname{Spec}(R)$ imply $\operatorname{Spec}(R) \cup \operatorname{Spec}(R) \neq \operatorname{Spec}(R)$.

(iii) If $2 \notin Z(R)$, then every vertex of $\Gamma(R)$ is a 4 -cycle vertex.

The next theorem will help to characterize all possible cycles in the idealbased zero-divisor graph. 
Theorem 3.9. Let $N$ be a 2-primal near-ring, $a, b \in \Gamma_{\mathbb{P}}(N)$ and $2 \notin \mathbb{P}$. If $2 \notin \Gamma_{\mathbb{P}}(N)$, then

(i) $c(a, b)=3$ if and only if $D(a) \cap D(b)=\phi$ and $\operatorname{Supp}(a) \cup \operatorname{Supp}(b) \neq$ $\operatorname{Spec}(N)$.

(ii) $c(a, b)=4$ if and only if either $D(a) \cap D(b) \neq \phi$ and $\operatorname{Supp}(a) \cup$ $\operatorname{Supp}(b) \neq \operatorname{Spec}(N)$, or $D(a) \cap D(b)=\phi$, and $\operatorname{Supp}(a) \cup \operatorname{Supp}(b)=$ $\operatorname{Spec}(N)$.

(iii) $c(a, b)=6$ if and only if $D(a) \cap D(b) \neq \phi$ and $\operatorname{Supp}(a) \cup \operatorname{Supp}(b)=$ $\operatorname{Spec}(N)$.

Proof. (i) Follows from Lemma 3.2 and Theorem 3.3.

(ii) If $D(a) \cap D(b)=\phi$ and $\operatorname{Supp}(a) \cup \operatorname{Supp}(b)=\operatorname{Spec}(N)$, there exists a path with vertices $a, b, 2 a$ and $2 b$, i.e., $c(a, b) \leq 4$. Now (i) implies that $c(a, b)=4$. If $D(a) \cap D(b) \neq \phi$ and $\operatorname{Supp}(a) \cup \operatorname{Supp}(b) \neq \operatorname{Spec}(N)$, then by Theorem 3.3, there exists $c \in \Gamma_{\mathbb{P}}(N)$ such that $c$ is adjacent to both $a$ and $b$. Thus, the path with vertices $a, c, b$ and $2 c$ is a cycle with length 4 .

(iii) If $c(a, b)=6$, then parts (i) and (ii) imply that $D(a) \cap D(b) \neq \phi$ and $\operatorname{Supp}(a) \cup \operatorname{Supp}(b)=\operatorname{Spec}(N)$. Conversely, let $D(a) \cap D(b) \neq \phi$ and $\operatorname{Supp}(a) \cup$ $\operatorname{Supp}(b)=\operatorname{Spec}(N)$. Then by Theorem 3.3, $d(a, b)=3$. Also, (i) and (ii) implies that $c(a, b)>4$. Hence, there are vertices $c$ and $d$ such that $a c, c d, b d \in \mathbb{P}$. Now, if some vertex $e$ is adjacent to $b$, then $b e \in \mathbb{P}$. Therefore, $\operatorname{Spec}(N)=$ $\operatorname{Supp}(a) \cup \operatorname{Supp}(b) \subseteq V(c) \cup V(e)$. However, $d(a, b)=3$ implies that $a$ is not adjacent to $e$, i.e., $c(a, b)=6$. If we consider the vertices $2 c$ and $2 d$, then we have a cycle with vertices $a, c, b, 2 d$ and $2 c$, i.e., $c(a, b)=6$.

From Theorem 3.9, we have the following corollary.

Corollary 3.10 ([11], Theorem 3.4). Let $R$ be a commutative reduced ring, $a, b \in \Gamma(R)$, and $2 \notin \Gamma(R)$. Then

(i) $c(a, b)=3$ if and only if $D(a) \cap D(b)=\phi$ and $\operatorname{Supp}(a) \cup \operatorname{Supp}(b) \neq$ $\operatorname{Spec}(R)$.

(ii) $c(a, b)=4$ if and only if either $D(a) \cap D(b) \neq \phi$ and $\operatorname{Supp}(a) \cup$ $\operatorname{Supp}(b) \neq \operatorname{Spec}(R)$ or $D(a) \cap D(b)=\phi$ and $\operatorname{Supp}(a) \cup \operatorname{Supp}(b)=$ $\operatorname{Spec}(R)$.

(iii) $c(a, b)=6$ if and only if $D(a) \cap D(b) \neq \phi$ and $\operatorname{Supp}(a) \cup \operatorname{Supp}(b)=$ $\operatorname{Spec}(R)$.

As an immediate application of Theorem 3.9 or Corollary 3.10 , we have the following corollary.

Corollary 3.11 ([11], Corollary 3.5). Let $R$ be a commutative reduced ring and $2 \notin \Gamma(R)$. Then every edge of a cycle with length 3 or 4 .

Proof. Let $a \approx b$ be an edge in a cycle. Then $a b \in \mathbb{P}$ and $D(a) \cap D(b)=\phi$. If $\operatorname{Supp}(a) \cup \operatorname{Supp}(b) \neq \operatorname{Spec}(R)$, then by Corollary 3.10, we have $c(a, b)=3$. Otherwise, $\operatorname{Supp}(a) \cup \operatorname{Supp}(b)=\operatorname{Spec}(R)$. Then by Corollary 3.10, we have $c(a, b)=4$. 
Acknowledgement. The second author is grateful to D. F. Anderson, Mathematics Department, The University of Tennesse, for his kind help. The authors express their sincere thanks to the referee for his/her valuable comments and suggestions which improve the paper a lot.

\section{References}

[1] D. D. Anderson and M. Naseer, Beck's coloring of a commutative ring, J. Algebra 159 (1993), no. 2, 500-514.

[2] D. F. Anderson and P. S. Livingston, The zero-divisor graph of a commutative ring, J. Algebra 217 (1999), no. 2, 434-447.

[3] I. Beck, Coloring of commutative rings, J. Algebra 116 (1988), no. 1, 208-226.

[4] J. A. Bondy and U. S. R. Murty, Graph Theory with Applications, American Elsevier Publishing Co., Inc., New York, 1976.

[5] P. Dheena and B. Elavarasan, On strong IFP near-ring, Submitted.

[6] R. Engelking, General Topology, Heldermann Verlag, Berlin, 1989.

[7] J. R. Munkres, Topology, Prentice-Hall of Indin, New Delhi, 2005.

[8] G. Pilz, Near-Rings, North-Holland, Amsterdam, 1983.

[9] S. P. Redmond, An ideal-based zero-divisor graph of a commutative ring, Comm. Algebra 31 (2003), no. 9, 4425-4443.

[10] K. Samei, On summand ideals in commutative reduced rings, Comm. Algebra 32 (2004), no. 3, 1061-1068.

[11] _ The zero-divisor graph of a reduced ring, J. Pure Appl. Algebra 209 (2007), no. 3, 813-821.

[12] S. H. Sun, Noncommutative rings in which every prime ideal is contained in a unique maximal ideal, J. Pure Appl. Algebra 76 (1991), no. 2, 179-192.

Patchirajulu Dheena

Department of Mathematics

ANNAMALAi UNIVERSITY

Annamalainagar - 608002, TAmilnadu, India

E-mail address: dheenap@yahoo.com

BALASUbramanian Elavarasan

Department of Mathematics

AnNamalai University

AnNAMAlainAgAR - 608002, TAMILnADU, INDIA

(PRESENT ADDRESS)

Department of Mathematics

School of Science And Humanities

KARUNYA UNIVERSITY

Coimbatore - 641114, TAmilnadu, India

E-mail address: belavarasan@gmail.com 